“(C) 2018 IEEE. Personal use of this material is permitted. Permission from IEEE must be obtained for all other uses, in any current or future media, including reprinting/republishing this material for advertising or promotional purposes, creating new collective works, for resale or redistribution to servers or lists, or reuse of any copyrighted component of this work in other works." 


\title{
Design and Analysis of a Wide Angle Impedance Matching Metasurface for Wideband Antenna Arrays
}

\author{
Alpha O. Bah ${ }^{1}$, Richard W. Ziolkowski ${ }^{1}$, Pei-Yuan Qin ${ }^{1}$, and Y. Jay Guo ${ }^{1}$ \\ ${ }^{1}$ Global Big Data Technologies Centre, University of Technology Sydney, New South Wales, 2007, Australia, \\ alpha.bah@student.uts.edu.au
}

\begin{abstract}
A wide bandwidth, low profile, double sided, wide angle impedance matching metasurface is reported. It alleviates the well-known problem of impedance mismatch caused by mutual coupling when an array is in its scan mode. Each unit cell of the metasurface contains two multi-resonant, tightly-coupled unequal arm Jerusalem cross elements on the top and bottom sides of a thin substrate. Each element consists of two orthogonal capacitively loaded strips. The wide bandwidth of the metasurface is achieved by tightly coupling these multi-resonant elements. The metasurface is capable of facilitating wide angle scanning over a 6:1 impedance bandwidth without the need for bulky dielectrics or multilayered structures.
\end{abstract}

Index Terms - Bandwidth, metasurfaces, phased arrays, tightly coupled arrays, wide angle impedance matching, wide bandwidth arrays.

\section{INTRODUCTION}

Recent developments in the fields of radar and satellite communications have highlighted the need for wideband multifunctional volumetrically scanned phased arrays [1]. This demand is brought about by the need for mobility while maintaining escalating data rate demands in both civilian and military applications. Phased arrays are capable of electronic beam scanning while remaining stationary. However, this attribute comes at the expense of increased loss at wider scan angles. The scan loss reduces the realized gain of the array. To minimize the reduction in the realized gain of a scanning array, wide angle impedance matching (WAIM) techniques have been used to address the mismatch at the aperture-air interface as well as that between the active impedance of the array and its feed lines.

Several WAIM schemes have been reported over the years. The earliest techniques involved using thin highdielectric-constant superstrates in front of an array aperture [3], placing a dielectric slab adjacent to the array aperture [45], and using tightly packed array elements [4]. The latter reduces to the Wheeler current sheet model [6] when the element separation approaches zero. These WAIM schemes have several drawbacks. They may increase the array height, improve scanning in one plane at the expense of the others, or increase the chances of introducing array blindness. Furthermore, they were limited to single frequency or narrow bandwidth operations.

More recently, metamaterials (MTMs) [7]-[10], frequency selective surfaces (FSSs) [11], and optimisation algorithms [12] have been used to provide WAIM functionalities to phased arrays. The constituent parameters of a MTM can be tuned to obtain electromagnetic properties that are not readily available in nature. This unique characteristic of MTMs was exploited [7] to realize an anisotropic WAIM structure for an array of open-ended circular waveguides. The resulting MTM-WAIM arrangement provided an improved match of the array to free space over several angles in comparison to an isotropic dielectric slab. Unfortunately, only the scanning results for the H-plane were presented; the associated E-plane and Dplane results were not reported. Moreover, wide angle scanning was the focus of the design, but it was achieved only over a $3.3 \%$ bandwidth.

The efforts reported in [3] and [7] were extended in [8]. The dielectric slab was replaced with an ultra-thin metasurface (MS) composed of subwavelength split-ring resonators (SRRs) for an improved scan in the $\mathrm{H}$ and $\mathrm{D}$ planes. The focus in [8] was also for wide angle scanning, but it again was achieved only at a single frequency.

While there have been some WAIM advances, it is again emphasised that most of the past work on wide angle scanning was achieved only over narrow bandwidths. In this paper, we propose a new lightweight, low profile, double sided metasurface (DS-MS) WAIM. The DS-MS has a near zero index (NZI) of refraction that can facilitate wide angle impedance matching in antenna arrays over a 6:1 impedance bandwidth without the need for bulky dielectrics or multilayered structures. Because of the NZI property of the DSMS, it can also serve as a wideband waveform transformer which can lead to increased directivity when used as a superstrate [13].

This paper is organized as follows. Section II introduces the structure and theory of operation of the DS-MS. Section III gives the design and analysis details of the DS-MS. The full wave simulation process for the DS-MS and the extraction of its effective parameters are detailed. Our conclusions and final remarks are presented in Section IV.

\section{METASURFACE STRUCTURE AND OPERATION}

The unit cell configuration of the DS-MS is shown in Fig. 1. Each element on the top and bottom surfaces of the DS-MS represent a tightly-coupled unequal arm Jerusalem cross (TC-UAJC). It consists of two orthogonal, bent-arm, capacitively-loaded-strips whose lengths are tuned to the desired operating frequencies. The arm segments of the element provide inductance and the gaps between the extremities of its bent arms provide capacitance. 


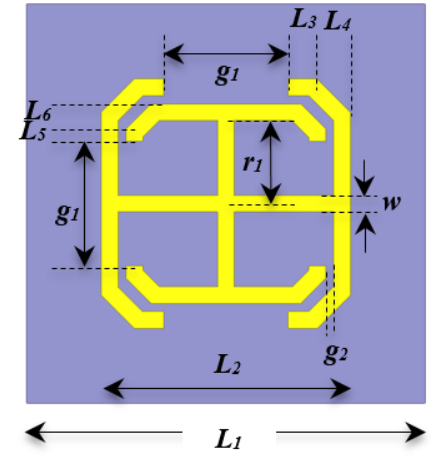

(a)

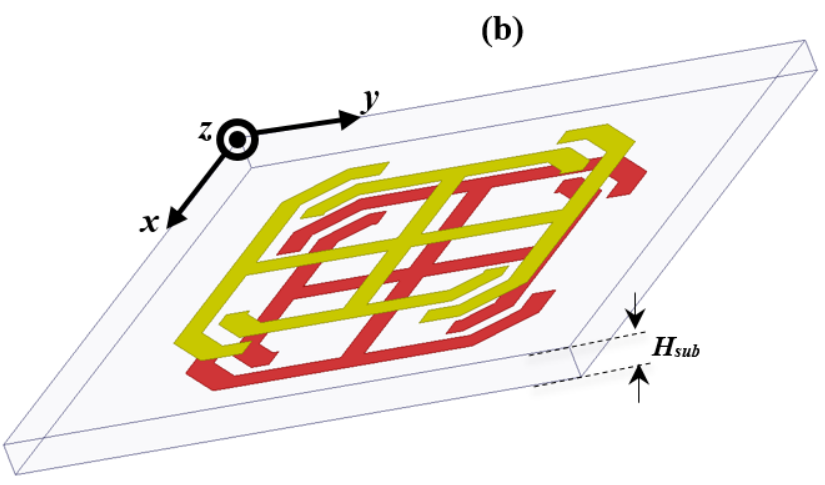

Fig. 1. DS-MS unit cell geometry. (a) Top view. (b) 3D view. The DS-MS unit cell dimensions in millimeters are: $\mathrm{w}=0.2, \mathrm{H}_{\text {sub }}=0.254, \mathrm{~g}_{1}=1.5, \mathrm{~g}_{2}=$ $0.1, \mathrm{~L}_{1}=4.8, \mathrm{~L}_{2}=3.0, \mathrm{~L}_{3}=0.35, \mathrm{~L}_{4}=0.4, \mathrm{~L}_{5}=0.15, \mathrm{~L}_{6}=0.3$, and $\mathrm{r}_{1}=1.0$. The substrate is Rogers RT/Duroid ${ }^{\mathrm{TM}} 5880$ with $\varepsilon_{\mathrm{r}}=2.2$ and $\tan \delta=0.0009$. The direction of propagation of the exciting wave is along the $\mathrm{z}$-axis.

The TC-UAJC elements are etched on the $17 \mu \mathrm{m}$ thick copper sheets that clad the top and bottom surfaces of a sheet of Rogers RT/Duroid ${ }^{\mathrm{TM}} 5880$ substrate with a relative dielectric constant $\varepsilon_{\mathrm{r}}=2.2$, loss tangent $\tan \delta=0.0009$, and a height of $0.254 \mathrm{~mm}$. The TC-UAJC elements on the bottom of the substrate are rotated $90^{\circ}$ relative to those on the top. This rotated arrangement of the top and bottom elements ensures stronger inter-element coupling within the unit cell and increased overall symmetry.

This TC-UAJC element employs several features to obtain the desired transmission and reflection characteristics required to achieve a wide bandwidth WAIM superstrate. The elements are closely spaced to increase their coupling capacitance. The horizontal and vertical components of the TC-UAJC are tightly coupled to each other to also increase the capacitance while their thin traces are used to increase the inductance. These properties facilitate both compactness and the realization of an inherently wideband element [14].

\section{METASURFACE SIMULATION AND ANALYSIS}

The design of the DS-MS was performed with ANSYSHFSS [15] using master-slave periodic boundary conditions in both the $x$ and $y$ directions of the unit cell depicted in Fig. 1. The zeroth-order Floquet modes, $\mathrm{TM}_{00}$ and $\mathrm{TE}_{00}$, were used for scanning in the $y-z$ and $x-z$ planes, respectively.
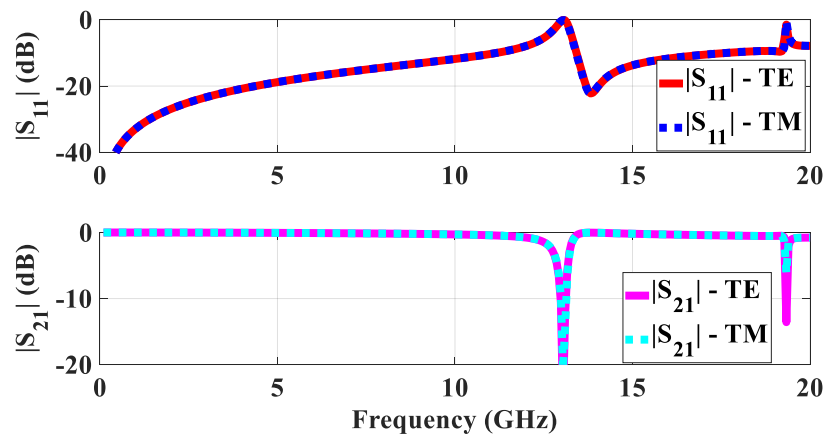

Fig. 2. Magnitudes of the S-parameters for the $\mathrm{TE}_{00}$ and $\mathrm{TM}_{00}$ excitations of the DS-MS. The first resonance frequency for both the $\mathrm{TE}_{00}$ and $\mathrm{TM}_{00}$ Floquet modes are at $13.04 \mathrm{GHz}$. There is also a second resonance frequency at $19.33 \mathrm{GHz}$.

The $y-z$ plane was taken to be the plane of incidence. The $\mathrm{TM}_{00}$ Floquet mode is parallel polarized, i.e., its electric field is along the y-direction and it propagates along the $\mathrm{z}$ direction, normal to the surface. The $\mathrm{TE}_{00}$ Floquet mode is perpendicularly polarized, i.e., it also propagates along the $\mathrm{z}$-direction, but with its electric field oriented along the $\mathrm{x}$ direction, transverse to the plane of incidence.

The DS-MS was simulated and an initial optimization was carried out to obtain the best transmission and reflection parameters possible. For the DS-MS to be successfully implemented as a wideband WAIM, its inter-element, intraelement, and radiator-MS coupling behaviours need to be re-adjusted to compensate for the impedance mismatch at the aperture-air interface.

The DS-MS possesses four-fold rotational symmetry and hence the TE and TM resonances coincide with each other. The identical TE and TM responses are displayed in Fig 2. We can see that the first reflection resonance frequency of the MS is above $13 \mathrm{GHz}$, which is much higher than the intended operational frequencies of the antenna array which are below 5.0 GHz. This outcome thus avoids operating in the highly dispersive and lossy resonance regions [7]. We can also see that the return loss for both the TE and TM polarizations is greater than $19 \mathrm{~dB}$ across the frequency band of interest and, hence, the insertion loss is virtually zero.

The transmission phase variation with frequency for the TM (parallel) polarized incident fields for various angles of incidence is shown in Fig. 3a. The phase varies by less than $4^{\circ}$ for a $0^{\circ}-70^{\circ}$ change in the incident angle. This small change in phase implies that the MS will facilitate the same output response for all of these incidence angles; and, thus, it can maintain polarization purity for its frequencies of operation. The phase variation actually improves with increasing incidence angles. These results suggest that if a radiator's E-plane is aligned with the specified TM plane of incidence, its scanning range will be improved because the maximum unit-cell-radiator coupling is along this plane. Consequently, when the DS- MS is used as a WAIM for a 
(a)

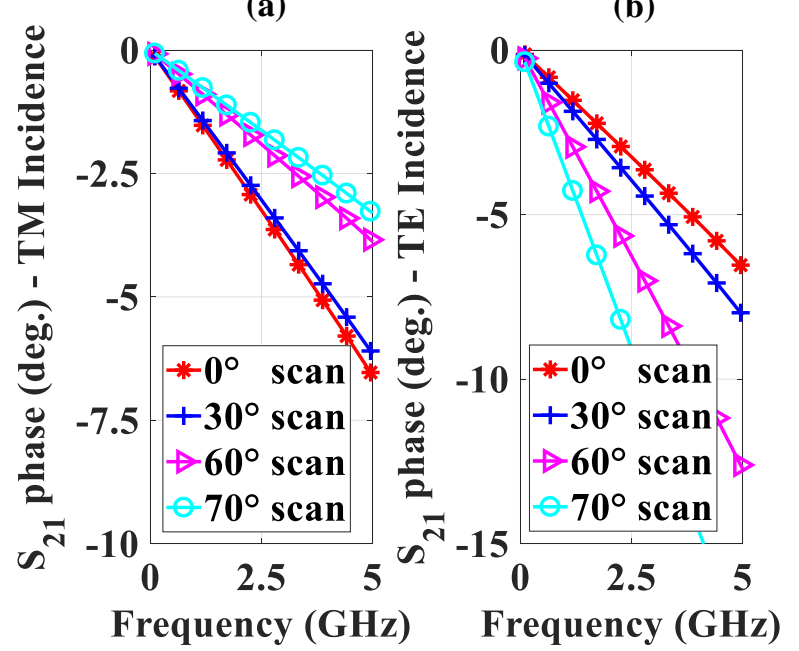

Fig. 3. Transmission phase variation with frequency for the different angles of incidence and polarizations. (a) TM polarization. (b) TE polarization.

ground plane backed antenna array, it can minimize the variation of the array's active reflection coefficient with scan angle. It does this by introducing a capacitive reactance and, hence, by acting as a wideband conjugate matching layer to counteract the effects of the ground plane's inductive reactance at wider scan angles [3].

For the TE (perpendicular) polarized incident fields, the phase varies by more than $10^{\circ}$ for a $0^{\circ}-70^{\circ}$ change in the incident angle as seen in Fig. 3b. The phase also deteriorates as the incident angle increases. Thus, aligning a radiator's E-plane with the TE polarized incident field plane (which is orthogonal to the specified plane of incidence) will not give rise to any improvements in the scanning range.

\section{A. Parameter extraction}

To fully characterize the DS-MS, its effective medium parameters were extracted. The method described in [16] was used to determine the effective wave impedance $Z_{\text {eff }}$, the effective refractive index $n_{\text {eff }}$, the effective dielectric constant $\varepsilon_{\text {eff }}$, and the effective permeability $\mu_{\text {eff }}$ from the normally incident scattering parameters. These medium parameters are shown in Fig. 4. In order to obtain more complete extraction results, the DS-MS was simulated to $30 \mathrm{GHz}$, well above its resonance frequencies.

Referring to the frequency range of interest, 0.0 to 5.0 $\mathrm{GHz}$, the extracted parameters reveal the following. The

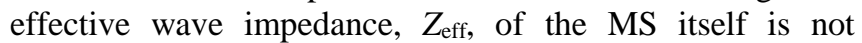
matched to free space. However, its parameters can be adjusted to enable it to act as a low loss impedance matching transformer between an array aperture and free space. Furthermore, the effective permeability, $\mu_{\mathrm{eff}}$, and, hence, the effective refractive index, $n_{\text {eff, }}$ are nearly zero across the frequency band of interest. This NZI layer can convert the fields radiated by an external antenna array into planar wave fronts as the wave emerges from the NZI medium. (a)
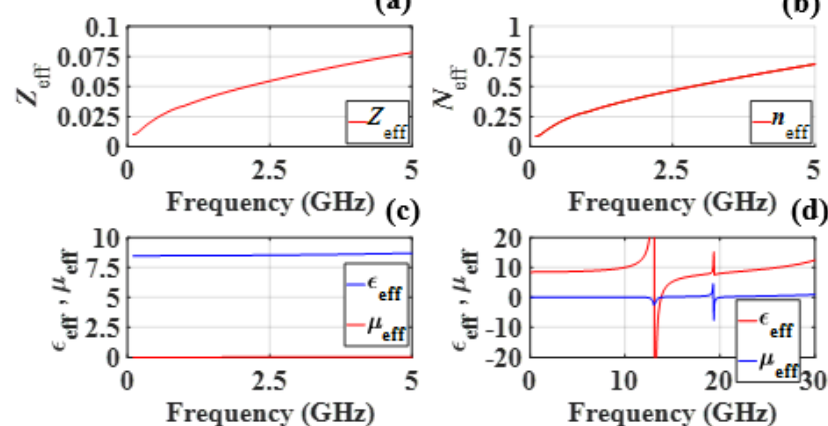

Fig. 4. Extracted parameters of the DS-MS for the TM excitation. (a) Effective impedance. (b) Effective refractive index. (c) Effective permittivity and effective permeability at the frequencies of interest and (d) across the whole band showing the two resonance frequencies at $13.04 \mathrm{GHz}$ and 19.33 GHz.

These planar wave fronts are a result of the spatially constant near-zero phase variation established within the DS-MS. Thus, the MS creates a uniform phase variation across its output face and, consequently, yields the highest possible directivity from its effective aperture [13]. Moreover, the values of $\varepsilon_{\text {eff }}$ and $\mu_{\text {eff }}$ are both essentially constant over the entire frequency band of interest which ensures a very desirable, dispersionless operation of the MS.

\section{CONCLUSION}

A wideband DS-MS to be used as a WAIM layer for an antenna array and to facilitate an improvement of its maximum directivity was designed and simulated. The effective medium parameters of the DS-MS were extracted to help explain its working principles. It was shown that this DS-MS features a wide bandwidth and is dispersionless over the desired frequency range of operation. As will be demonstrated in our presentation, this DS-MS-based WAIM layer improves the scanning range of an antenna array over its wide bandwidth when it is employed as a superstrate.

\section{ACKNOWLEDGMENT}

This work is supported by the Australian Government Research Training Program Scholarship, the Cooperate Research Centre for Space Environment Management (SERC Limited) through the Australian Government's Coorporate Research Centre Program, and the Australia Research Council Discovery Program under Grant DP160102219.

\section{REFERENCES}

[1] G. C. Tavik, C. L. Hilterbrick, J. B. Evins, J. J. Alter, J. G. Crnkovich, J. W. de Graaf, W. Habicht, G. P. Hrin, S. A. Lessin, D. C. Wu, and S. M. Hagewood, "The advanced multifunction RF concept," IEEE Trans. Microw. Theory Techn., vol. 53, no. 3, pp. 1009-1020, Mar. 2005. 
[2] A. Oliner and G. Knittel, Eds., "Phased array antennas," in Proc. 1970 Phased Array Symposium, 1972 (Artech House, Dedham, MA).

[3] E. G. Magill and H. A. Wheeler, "Wide-angle impedance matching of a planar array antenna by a dielectric sheet," IEEE Trans. Antennas Propag., vol. 14, no. 1, pp. 49-53, Jan. 1966.

[4] V. Galindo and C. P. Wu, "Dielectric loaded and covered rectangular waveguide phased arrays," Bell Syst. Tech. J., vol. 47, no. 1, pp. 93-116, Jan. 1968.

[5] S. Lee and R. Mittra, "Radiation from dielectric-loaded arrays of parallel-plate waveguides," IEEE Trans. Antennas Propag., vol. 16, no. 5, pp. 513-519, Sep. 1968.

[6] H. A. Wheeler, "Simple relations derived from a phased-array antenna made of an infinite current sheet," IEEE Trans. Antennas Propag., vol. 13, no. 4, pp. 506-514, Jul. 1965.

[7] S. Sajuyigbe, M. Ross, P. Geren, S. A. Cummer, M. H. Tanielian, and D. R. Smith, "Wide angle impedance matching metamaterials for waveguide-fed phased-array antennas," IET Microw. Antennas Propag., vol. 4, no. 8, pp. 1063-1072, Aug. 2010.

[8] T. R. Cameron and G. V. Eleftheriades, "Analysis and characterization of a wide-angle impedance matching metasurface for dipole phased arrays," IEEE Trans. Antennas Propag., vol. 63, no. 9, pp. 3928-3938, Sep. 2015.

[9] F. Silvestri, P. Chiusolo, L. Cifola, R. Bolt, G. Gerini, "Design of metamaterial based wide angle impedance matching layers for active phased arrays," in Proc. 9th European Conference on Antennas and Propagation (EuCAP 2015), Lisbon, 2015.

[10] P. Rodríguez-Ulibarri1, T. Crépin, C. Martel, F. Boust, F. Falcone, C. Loecker, K. Herbertz, T. Bertuch, T. Dousset, J.-P. Martinaud, S. Maci, J. A. Marcotegui, and M. Beruete, "Experimental demonstration of metamaterials application for mitigating scan blindness in phased array antennas," EPJ Appl. Metamat., vol. 3, 9, Oct. 2016.

[11] E. Yetisir, N. Ghalichechian and J. L. Volakis, "Ultrawideband array with $70^{\circ}$ scanning using FSS superstrate," IEEE Trans. Antennas Propag., vol. 64, no. 10, pp. 4256-4265, Oct. 2016.

[12] G. Oliveri, F. Viani, N. Anselmi, and A. Massa, "Synthesis of multilayer WAIM coatings for planar phased arrays within the system-by-design framework," IEEE Trans. Antennas Propag., vol. 63, no. 6, pp. 2482-2496, Jun. 2015.

[13] R. W. Ziolkowski, "Propagation in and scattering from a matched metamaterial having a zero index of refraction," Phys. Rev. E, vol. 70, no. 4, 046608, Oct. 2004.

[14] D. Schurig, J. J. Mock and D. R. Smith, "Electric-field-coupled resonators for negative permittivity metamaterials," in Applied Physics Letters, vol. 88, no. 4, 041109, 2006.

[15] ANSYS HFSS ver. 16, ANSYS, Inc., Canonsburg, PA [Online]. Available: https://www.ansoft.com/products/hf/hfss/

[16] Z. Szabo, G. H. Park, R. Hedge and E. P. Li, "A unique extraction of metamaterial parameters based on Kramers-Kronig relationship," IEEE Trans. Microw. Theory Techn., vol. 58, no. 10, pp. 2646-2653, Oct. 2010. 